\title{
Induction of tumor inhibitory anti-angiogenic response through immunization with interferon Gamma primed placental endothelial cells: ValloVax ${ }^{\mathrm{TM}}$
}

Thomas E Ichim ${ }^{1 *}{ }^{*}$, Shuang $\mathrm{Li}^{2+}{ }^{1}$, Hong Ma ${ }^{1}$, Yuliya V Yurova ${ }^{3}$, Julia S Szymanski ${ }^{1}$, Amit N Patel ${ }^{4}$, Santosh Kesari ${ }^{5,6}$, Wei-Ping Min $^{7}$ and Samuel C Wagner ${ }^{1}$

\begin{abstract}
Background: While the concept of angiogenesis blockade as a therapeutic intervention for cancer has been repeatedly demonstrated, the full promise of this approach has yet to be realized. Specifically, drugs such as VEGF-blocking antibodies or kinase inhibitors suffer from the drawbacks of resistance development, as well as off-target toxicities. Previous studies have demonstrated feasibility of specifically inducing immunity towards tumor endothelium without consequences of systemic autoimmunity in both animal models and clinical settings.

Method: Placenta-derived endothelial cells were isolated and pretreated with interferon gamma to enhance immunogenicity. Syngeneic mice received subcutaneous administration of B16 melanoma, 4 T1 mammary carcinoma, and Lewis Lung Carcinoma (LLC), followed by administration of control saline, control placental endothelial cells, and interferon gamma primed endothelial cells (ValloVax $\left.{ }^{\mathrm{TM}}\right)$. Tumor volume was quantified. An LLC metastasis model was also established and treated under similar conditions. Furthermore, a safety analysis in non-tumor bearing mice bracketing the proposed clinical dose was conducted.
\end{abstract}

Results: ValloVax ${ }^{\mathrm{TM}}$ immunization led to significant reduction of tumor growth and metastasis as compared to administration of non-treated placental endothelial cells. Mitotic inactivation by formalin fixation or irradiation preserved tumor inhibitory activity. Twenty-eight day evaluation of healthy male and female mice immunized with ValloVax ${ }^{\mathrm{TM}}$ resulted in no abnormalities or organ toxicities.

Conclusion: Given the established rationale behind the potential therapeutic benefit of inhibiting tumor angiogenesis as a treatment for cancer, immunization against a variety of endothelial cell antigens may produce the best clinical response, enhancing efficacy and reducing the likelihood of the development of treatment resistance. These data support the clinical evaluation of irradiated ValloVax ${ }^{\mathrm{TM}}$ as an anti-angiogenic cancer vaccine.

\section{Background}

Tumors utilize a variety of molecular mechanisms to evade the immune response, including loss of tumor specific antigens [1-3], suppression of antigen presenting machinery such as transporter associated protein and MHC expression [4-7], and the production of immunosuppressive factors, both soluble and surface bound [8]. Additionally,

\footnotetext{
* Correspondence: thomas.ichim@gmail.com

${ }^{\dagger}$ Equal contributors

'Batu Biologics Inc, San Diego, 9255 Towne Centre Drive, Suite 450, San Diego, CA 92121, USA

Full list of author information is available at the end of the article
}

tumors lack expression of co-stimulatory molecules critical for the activation of naïve T cells, and suppress the expression of these molecules on antigen presenting cells [9]. Tolerogenic means elaborated by the tumor inhibit $\mathrm{T}$ cell activation while creating a microenvironment conducive to T cell exhaustion. Poor T cell function in the tumor microenvironment allows tumors to escape immune-mediated destruction promoting the developent of treatment resistance through immunoediting [10]. The ability of tumors to escape immune pressure and sculpt their immunogenic phenotype to evade immune destruction makes it

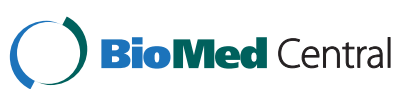

(c) 2015 Ichim et al.; licensee BioMed Central. This is an Open Access article distributed under the terms of the Creative Commons Attribution License (http://creativecommons.org/licenses/by/4.0), which permits unrestricted use, distribution, and reproduction in any medium, provided the original work is properly credited. The Creative Commons Public Domain Dedication waiver (http://creativecommons.org/publicdomain/zero/1.0/) applies to the data made available in this article, unless otherwise stated. 
exceedingly difficult to develop effective immunotherapies targeting tumor-derived antigens. A novel approach towards inducing anti-tumor immunity would be to target not the tumor itself, but the blood supply feeding the tumor, an essential mechanism of tumor growth.

Immunological targeting of tumor endothelium is appealing based on: a) For every tumor endothelial cell therapeutically neutralized approximately 200-300 tumor cells perish, thus reducing ability of tumors to lose expression of antigens; b) The immune system is in direct contact with the tumor endothelium, while immune access inside tumors is difficult due to areas of necrosis and high interstitial pressure; and c) Demonstrated prior efficacy of other anti-angiogenesis inhibitory compounds such as bevacizumab $[11,12]$. Furthermore, the elevated expression of Fas Ligand on the tumor endothelium mediates the selective killing of CD8+ Tumor Infiltrating Lymphocytes (TIL) allowing for a predominance of FoxP3+ T regulatory cells (Treg) to infiltrate the tumor microenvironment, demonstrating that the tumor blood vessels act as an immunological barrier promoting tumor tolerance [13]. Immune-mediated destruction of the tumor endothelium has been shown to significantly increase TILs in mouse models, which was correlated with tumor regression [14]. Another further potential benefit of targeting the tumor associated vasculature is the potential of sensitizing tumors to radiotherapy [15], in part due to the selective thrombotic and apoptotic effects irradiation has on the tumor vasculature [16-19]. Current tyrosine kinase inhibitors blocking angiogenesis systemically inhibit proangiogenic factors such as Vascular Endothelial Growth Factor (VEGF) or Angiopoetin, slowing blood vessel formation without differentiating between tumor and healthy angiogenesis. However, therapeutics that stimulate direct damage to the tumor endothelium have been shown to activate the coagulation cascade, effectively cutting off blood supply to the tumor and creating a hypoxic microenvironment conducive to necrosis and tumor regression [20]. A more effective anti-angiogenesis approach may be to stimulate selective killing of the tumor endothelium through immunotherapeutic vaccines.

A fundamental question determining feasibility of vaccineinduced killing of tumor vasculature is whether antigens exist on the tumor endothelium that are not expressed on physiologically normal blood vessels, and whether immunity could be raised against such antigens. A few tumor endothelium-specific antigens have been reported. The roundabout receptor (ROBO) -4 is a transmembrane protein that was originally found to orchestrate the neuronal guidance mechanism of the nervous system [21]. ROBO4 was found to be selectively expressed on tumor endothelial cells but not healthy vasculature [22]. Zhuang et al. demonstrated that mice immunized with the extracellular domain of mouse Robo4, showed a strong antibody response to Robo4, with no objectively detectable adverse effects on health, including normal menstruation and wound healing. Robo4 vaccinated mice showed impaired fibrovascular invasion and angiogenesis in a rodent sponge implantation assay, as well as a reduced growth of implanted syngeneic Lewis lung carcinoma. The antitumor effect of Robo4 vaccination was present in CD8 deficient mice but absent in B cell or IgG1 knockout mice, suggesting antibody-dependent cell mediated cytotoxicity as the anti-vascular/anti-tumor mechanism [23]. Another antigen that is more ubiquitously found throughout the body, but with higher expression on tumor endothelial cells is the VEGF receptor 2 (VEGFR2) which is typically found on hematopoietic stem cells and endothelial progenitor cells [24-29]. Despite expression on nonmalignant tissue, successful induction of antitumor immunity has been demonstrated using various immunization means against this antigen. Yan et al. utilized irradiated AdVEGFR2-infected cell vaccine-based immunotherapy in the weakly immunogenic and highly metastatic $4 \mathrm{~T} 1$ murine mammary cancer model. Lethally irradiated, virus-infected 4 T1 cells were used as vaccines. Vaccination with lethally irradiated AdVEGFR2-infected 4 T1 cells inhibited subsequent tumor growth and pulmonary metastasis compared with challenge inoculations. Angiogenesis was inhibited, and the number of CD8+ T lymphocytes was increased within the tumors. Antitumor activity was also caused by the adoptive transfer of isolated spleen lymphocytes, thus demonstrating induction of tumor specific immunity [30]. Other approaches have been utilized to induce immunity to VEGFR2, which resulted in induction of tumor regression without systemic toxicities [31-36]. Tumor endothelial marker 1 or endosialin is another antigen found selectively on the tumor vasculature. Facciponte et al. demonstrated that a DNA vaccination targeting endosialin reduced tumor vascularity, increased CD3+ T cell infiltration, and was correlated with significant inhibition of tumor growth. Epitope spreading to tumor antigens following the initial immune response against the tumor vasculature gives evidence that targeting the tumor endothelium may activate a cascade of pathways conducive to tumor regression. Additionally, the DNA vaccination against endosialin did not affect other angiogenesis dependent physiological processes, exhibiting no adverse effects on menstruation, embryonic development, pregnancy, and wound healing in mouse models [14]. Other markers associated with tumor blood vessels have been utilized therapeutically in animal models for vaccination purposes including survivin [37-39], xenogeneic FGF2R [40], VEGF [41], VEGF-R2 [42], MMP-2 [43], and endoglin $[44,45]$.

Although tumor endothelial cells are more genetically stable then the tumor cells, thus reducing the possibility of immune mediated antigen loss, some mutational activity 
has been reported in tumor associated vascular cells $[46,47]$. Accordingly, a polyvalent vaccine approach targeting the immune system toward a plethora of endothelial cell antigens specific to the tumor endothelium may be more effective. With this approach comes a heightened theoretical risk of autoimmunity. Despite these theoretical concerns successful immunization against tumor endothelium has been performed utilizing Human Umbilical Vein Endothelial Cells (HUVEC). Wei et al. demonstrated that vaccination of mice with fixed xenogeneic whole endothelial cells (in the form of HUVEC) as a vaccine was effective in affording protection from tumor growth, inducing regression of established tumors, and prolonging the survival of tumor-bearing mice. Additionally, the authors found that, immunity targeted to tumor vasculature was induced and was responsible for the anti-tumor activity, which was not associated with any noticeable toxicity toward non-malignant tissues $[48,49]$. From a clinical perspective, a 17 patient trial demonstrated that HUVEC vaccine therapy significantly prolonged tumor doubling time and inhibited tumor growth in patients with recurrent glioblastoma, inducing both cellular and humoral responses against the tumor vasculature without any adverse events or noticeable toxicities [50]. The clinical efficacy of using HUVEC vaccination to break tolerance to tumor angiogenesis has also been demonstrated in patients with colorectal cancer and malignant brain tumors without any observable adverse effects on healthy angiogenesis [20].

In this current study it was demonstrated that placental endothelial cells that are interferon gamma primed potently inhibit tumor growth in 3 histologically distinct animal models, as well as suppress pulmonary metastasis subsequent to intravenous tumor administration. Furthermore, the therapeutic effect was retained when placental endothelial cells have been mitotically inactivated by either formalin or irradiation. The success of this new approach may provide a new way to develop clinical effective placental cells vaccination against a wide variety of tumors by targeting tumor angiogenesis.

\section{Materials and methods}

\section{Animals and cells}

Female C57BL/6 and BALB/c mice aged 8-12 weeks were purchased from The Jackson Laboratory. Animals were housed under conventional conditions at the Animal Care Facility, University of Western Ontario, and were cared for in accordance with the guidelines established by the Canadian Council on Animal Care. A murine melanoma cell line established from a C57BL/6 mouse and designated B16F10 was obtained from the American Type Culture Collection (ATCC) and was maintained in RPMI 1640 medium (Sigma-Aldrich) with 10\% FBS, lglutamine, penicillin, and streptomycin at $37^{\circ} \mathrm{C}$ in $5 \%$
$\mathrm{CO}_{2}$. The murine mammary carcinoma $4 \mathrm{~T} 1$ cells (ATCC) were grown DMEM medium (Sigma-Aldrich) with 10\% FBS, l-glutamine, penicillin, and streptomycin at $37^{\circ} \mathrm{C}$ in $5 \% \mathrm{CO}_{2}$. Lewis Lung Carcinoma (LLC) is a murine lung carcinoma originating from C57/BL6 mice. The cells were maintained in RPMI 1640 supplemented with 10\% fetal bovine serum, $2 \mathrm{mM}$ glutamine (Gibco-BRL, Life Technologies, Inc.). The cell line was cultured at $37^{\circ} \mathrm{C}$ in a $5 \%$ incubator.

\section{Preparation of vaccine}

Full term human placentas were collected from delivery room under informed consent. Fetal membranes were manually peeled back and the villous tissue is isolated from the placental structure. Villous tissue was subsequently washed with cold saline to remove blood and scissors used to mechanically digest the tissue. Lots of 25 grams of minced tissue were incubated with approximately $50 \mathrm{ml}$ of HBSS with $25 \mathrm{mM}$ of HEPES and $0.28 \%$ collagenase, $0.25 \%$ dispase, and $0.01 \%$ DNAse at 37 Celsius. The mixture of minced placental villus tissue and digesting solution was incubated under stirring conditions for three incubation periods of 20 minutes each. Ten minutes after the first incubation period and immediately after the second and third incubation periods, the DNAse was added to make up a total concentration of DNase, by volume, of $0.01 \%$. In the first and second incubations, the incubation flask is set at an angle, and the tissue fragments allowed to settle for approximately 1 minute, with $35 \mathrm{ml}$ of the supernatant cell suspension being collected and replaced by $38 \mathrm{ml}$ (after the first digestion) or $28 \mathrm{ml}$ (after the second digestion) of fresh digestion solution. After the third digestion the whole supernatant was collected. The supernatant collected from all three incubations was then pooled and is poured through approximately four layers of sterile gauze and through one layer of 70 micrometer polyester mesh. The filtered solution was then centrifuged for $1000 \mathrm{~g}$ for 10 minutes through diluted new born calf serum, said new born calf serum diluted at a ratio of 1 volume saline to 7 volumes of new born calf serum. The pooled pellet was then resuspended in $35 \mathrm{ml}$ of warm DMEM with $25 \mathrm{mM}$ HEPES containing $5 \mathrm{mg}$ DNase I. The suspension was subsequently mixed with $10 \mathrm{ml}$ of $90 \%$ Percoll to give a final density of $1.027 \mathrm{~g} / \mathrm{ml}$ and centrifuged at $550 \mathrm{~g}$ for 10 minutes with the centrifuge brake off. The pellet was then washed in HBSS and cells incubated for 48 hours in complete DMEM media. After 3-4 passages cells were incubating in media containing $100 \mathrm{IU}$ of IFN-gamma per ml. Subsequent to incubation cells were either used: a) unmanipulated; b) used as a lysate, with 10 freeze thaw cycles in liquid nitrogen, subsequent to which lysate was filtered through a 0.2 micron filter; c) mitotically inactivated by irradiation at $10 \mathrm{~Gy}$; or d) inactivated by fixation in $0.5 \%$ formalin and subsequently washed. 
Immunization schedules and tumor assessment

For induction of tumor growth, $5 \times 10^{5} \mathrm{~B} 16$, LLC, or $4 \mathrm{~T} 1$ cells, American Type Culture Collection (Manassas, VA) cells were injected subcutaneously into the hind limb flank. Four weekly vaccinations of $5 \times 10^{5}$ test cells were administered subcutaneously on the contralateral side to which tumors were administered. Vaccination was performed on the day of tumor inoculation and on days 7, 14, and 21. Tumor growth was assessed every 3 days by two measurements of perpendicular diameters by a caliper, and animals were sacrificed when tumors reached a size of $1 \mathrm{~cm}$ in any direction. Tumor volume was calculated by the following formula: (the shortest diameter ${ }^{2} \times$ the longest diameter) $/ 2$.

\section{Results}

Successful utilization of placental endothelial cells in induction of anticancer immunity regardless of tumor type

While it has previously been demonstrated that vaccination with autologous and allogeneic endothelial cells results in tumor regression [51-55], and safety of this approach has been reported in clinical studies [20], current means of extracting endothelial cells are limited to the need for tissue culture expansion. Generally endothelial cells proliferate poorly in vitro and require the addition of recombinant growth factors that add expense, as well as possibility of contamination during production of clinical grade production. Accordingly, a more practical source of endothelium would be the placental body, which contains up to 2-10 billion primary endothelial cells per placenta [56-58]. We immunized mice bearing LLC, B16 and 4 T1 cells. The immunization schedule was a therapeutic one in that time of immunization occurred concurrently with the administration of tumor challenge. As seen in Figures 1, 2 and 3, a trend towards reduction of tumor growth was observed with non-IFN-gamma pretreated endothelial cells, while a potent reduction of tumor growth was seen in animals treated with cells that were first stimulated with interferon gamma. Interferon gamma pretreatment was shown to upregulate HLA I and HLA II (data not shown).

\section{Mitotically inactivated endothelial cell vaccine retains antitumor activity across histologically different tumors}

For clinical development of a cancer angiogenesis vaccine, it is imperative to generate cells that are mitotically inactivated. Part of the reason for this is that administration of viable endothelial cells could potentially result in acceleration of tumor growth through enhancement of angiogenesis [59]. Furthermore, in previous clinical trials, endothelial cells were pretreated with a fixative to avoid this potential issue [20]. As seen in Figures 4, 5 and 6 , for all tumor models tested, mitotic inactivation utilizing irradiation was mildly superior to formalin fixation. Additionally, no therapeutic effect was observed by administration of endothelial cell lysate, with activity being retained in activated endothelial cells.

\section{Endothelial cell vaccine inhibit tumor metastasis}

In addition to reduction of tumor size growth, an important aspect of cancer immunization is reduction in lung metastasis. Importantly, in the utilization of vaccines against cancer, the ability of the immune system to seek and destroy metastatic cells is one of the key enticing factors of this approach. When intravenous administration of LLC was performed in C57BL/6 mice, immunization with ValloVax ${ }^{\mathrm{Tm}}$ resulted in inhibition of tumor lung metastasis colonies (Figure 7). The inhibition

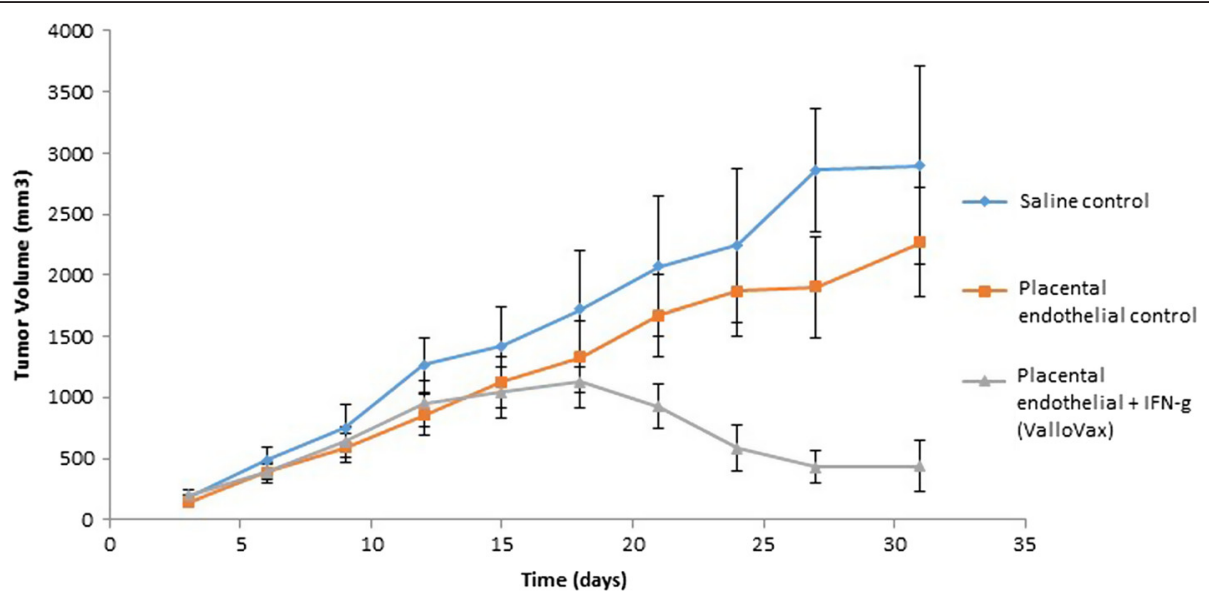

Figure 1 ValloVax ${ }^{\text {TM }}$ treatment inhibits B16 melanoma growth. Female C57BL/6 mice (10 mice per group) were immunized with saline (diamond), or $5 \times 10(5)$ placental endothelium cells (square) or placental endothelium cells pretreated with interferon gamma (triangle) on days 0 , 7, 14, and 21. Tumor growth was initiated by subcutaneous administration of $5 \times 10(5)$ B16 cells and quantified every third day. 


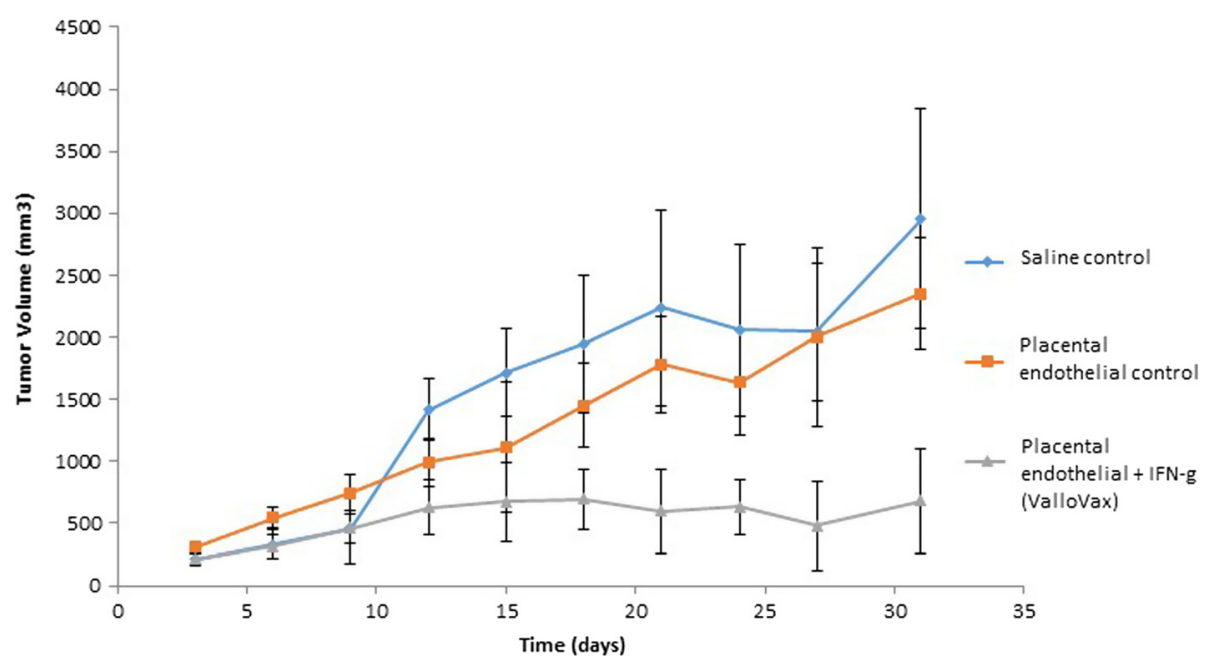

Figure 2 ValloVax ${ }^{\mathrm{TM}}$ treatment inhibits $4 \mathrm{~T} 1$ mammary carcinoma growth. Female BALB/C mice (10 mice per group) were immunized with saline (diamond), or $5 \times 10(5)$ placental endothelium cells (square) or placental endothelium cells pretreated with interferon gamma (triangle) on days $0,7,14$, and 21 . Tumor growth was initiated by subcutaneous administration of $5 \times 10(5) 4 \mathrm{~T} 1$ cells and quantified every third day.

was significantly more profound when placental endothelial cells were treated with interferon gamma.

\section{Safety evaluation}

Seven male and female mice per group were treated with control, or 500,000, 2 million or 4 million ValloVax ${ }^{\mathrm{Tn}}$ irradiated cells subcutaneously. Cells were administrated as in the therapeutic protocol, in that they were given on day $0,7,14$, and 21 . Body weights were calculated every three days and organ sizes, biochemical and hematological parameters were evaluated. No significant deviation was noted, nor were signs of autoimmunity present (online Additional file 1). These preclinical data support the safety of the irradiated ValloVax ${ }^{\mathrm{mi}}$ approach.

\section{Discussion}

The concept of targeting tumor associated endothelium has been a holy grail of cancer therapists since the original work of Judah Folkman demonstrated that tumors cannot grow more than 1-2 millimeters without the stimulation of new blood vessel formation (angiogenesis) [60]. Specifically blocking angiogenesis is intellectually enticing because the tumor-associated endothelium is derived from non-mutated tissue; therefore the possibility of development of a drug resistant phenotype is very low. Unfortunately several angiogenesis-targeting drugs that have demonstrated promising results in animal trials have failed in pivotal clinical trials. Examples include angiostatin, endostatin, and shark cartilage extract (Neovastat) [61,62].

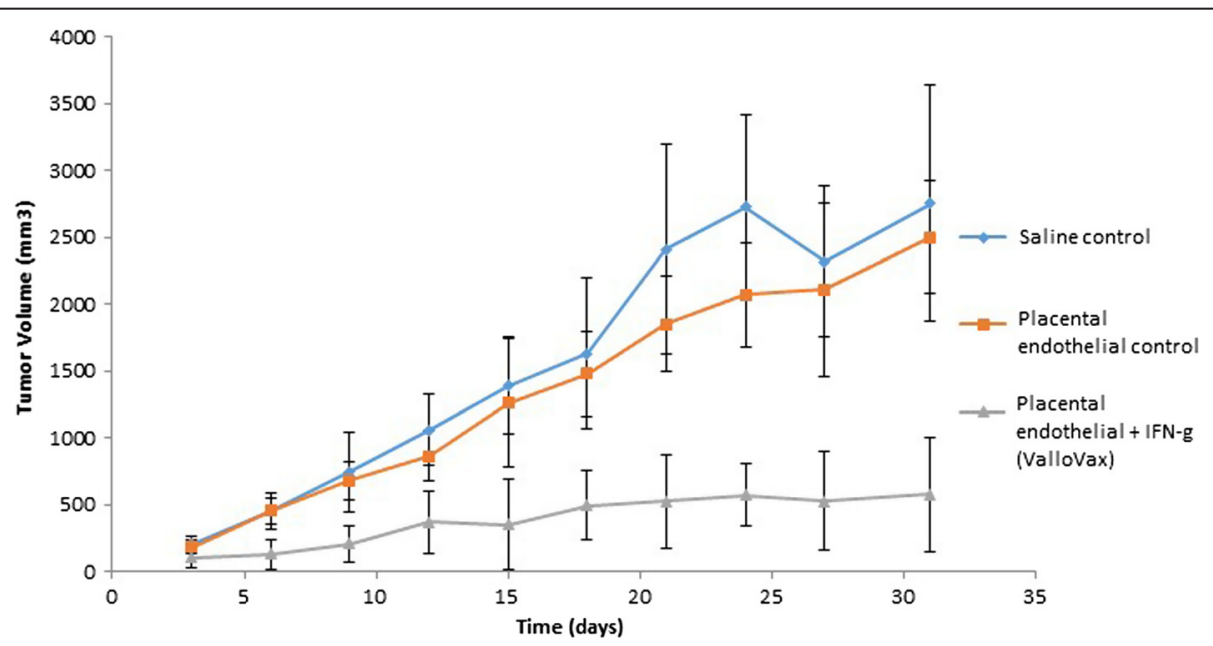

Figure 3 ValloVax $^{\mathrm{TM}}$ treatment inhibits LCC lung carcinoma growth. Female C57BL/6 mice (10 mice per group) were immunized with saline (diamond), or $5 \times 10$ (5) placental endothelium cells (square) or placental endothelium cells pretreated with interferon gamma (triangle) on days 0 , 7, 14, and 21. Tumor growth was initiated by subcutaneous administration of $5 \times 10$ (5) LLC cells and quantified every third day. 


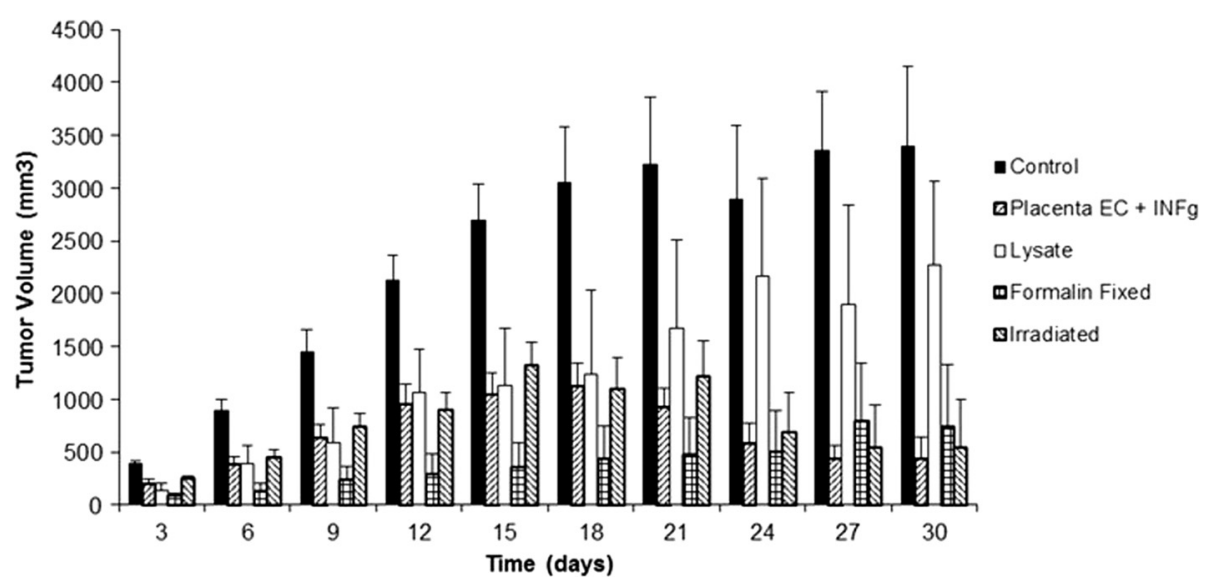

Figure 4 Mitotically inactivated ValloVax ${ }^{\mathrm{TM}}$ retains activity against B16 melanoma growth. Female C57BL/6 mice (10 mice per group) were immunized with saline, ValloVax ${ }^{\mathrm{TM}}$, ValloVax ${ }^{\mathrm{TM}}$ lysate, formalin fixed ValloVax ${ }^{\mathrm{TM}}$, or irradiated Vallovax ${ }^{\mathrm{TM}}$ at a concentration of $5 \times 10(5)$ cells or cell equivalents on days $0,7,14$, and 21. Tumor growth was initiated by subcutaneous administration of $5 \times 10(5)$ B16 cells and quantified every third day.

More recent studies using the VEGF pathway blocking antibody, bevacizumab (Avastin), have demonstrated positive results in specific types of tumors, which led to regulatory approvals [63]. Unfortunately the use of these antibodies requires co-administration of chemotherapy, and is associated with signification toxicity [64]. Furthermore, for reasons unknown, bevacizumab is ineffective in several tumor types, and in the tumors that it is effective, resistance often ensues, limiting long-term therapeutic utility [65].

The possibility of inducing selective immunity to proliferating blood vessels has been previously reported in animal models as well as pilot clinical trials. Unfortunately, a major limiting factor to clinical implementation has been the utilization of HUVEC cells as an antigenic source, which is limited in availability. Here we utilized placental derived endothelial cells as a source of antigen found on proliferating endothelium such as the tumor. The utilization of placenta derived tissues for immunization to cancer was initially introduced in the 1970 s by Dr. Valentin Govallo (reviewed in Harandi [66]) who demonstrated that immunity to placental trophoblast extract resulted in reduction of immune suppression using the PHA stimulation assay, as well as radiological tumor reductions. Dr. Govallo noted the immunological similarities between pregnancy and cancer. Later along with the advancement in molecular biology development founding placental cells and tumor endothelial cells share the molecules of angiogenesis such as VEGF, placental growth factor, angiopoietin, FGF, EGF, and TGF-beta, and as well placental endothelium expresses many of the novel tumor endothelial markers (TEM) such as ROBO4 [67], CLEC14A [68],

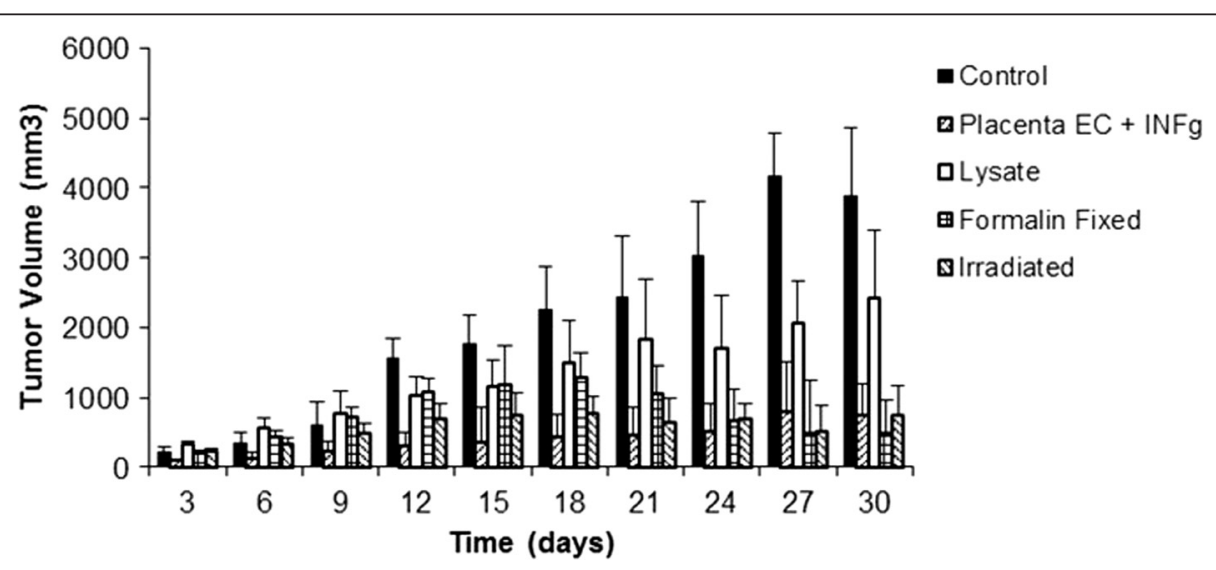

Figure 5 Mitotically inactivated ValloVax ${ }^{\mathrm{TM}}$ retains activity against $4 \mathrm{~T} 1$ mammary carcinoma growth. Female BALB/C mice (10 mice per group) were immunized with saline, ValloVax ${ }^{\mathrm{T}}$, ValloVax ${ }^{\mathrm{TM}}$ lysate, formalin fixed ValloVax ${ }^{\mathrm{TM}}$, or irradiated Vallovax ${ }^{\mathrm{TM}}$ at a concentration of $5 \times 10(5)$ cells or cell equivalents on days $0,7,14$, and 21 . Tumor growth was initiated by subcutaneous administration of $5 \times 10(5) 4 \mathrm{~T} 1$ cells and quantified every third day. 


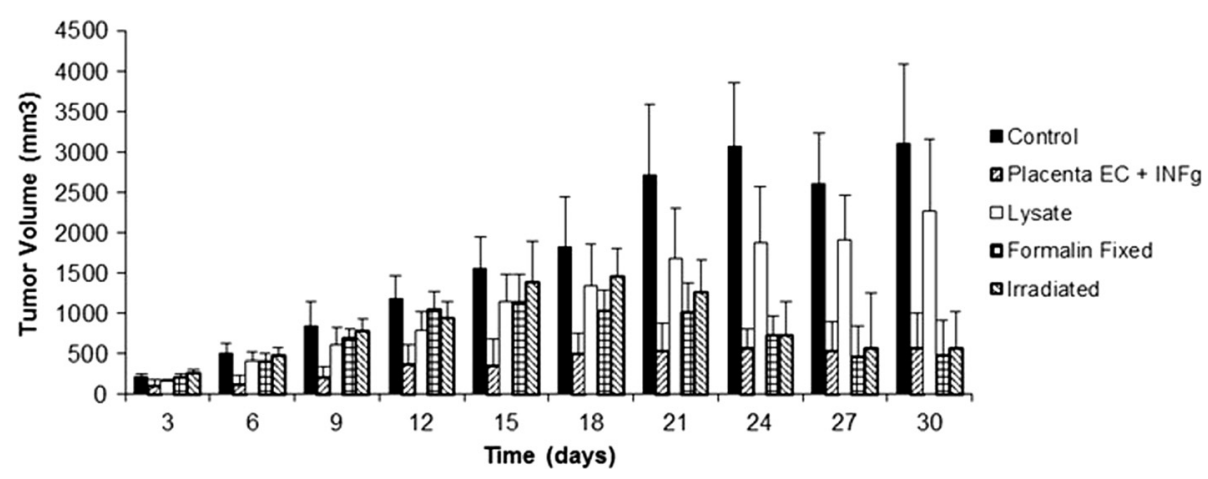

Figure 6 Mitotically inactivated ValloVax ${ }^{\mathrm{Tm}}$ Retains $^{\mathrm{Tm}}$ activity against LLC lung carcinoma. Female C57BL/6 mice (10 mice per group) were immunized with saline, ValloVax ${ }^{\mathrm{TM}}$, ValloVax ${ }^{\mathrm{TM}}$ lysate, formalin fixed ValloVax ${ }^{\mathrm{TM}}$, or irradiated Vallovax ${ }^{\mathrm{TM}}$ at a concentration of $5 \times 10(5)$ cells or cell equivalents on days $0,7,14$, and 21 . Tumor growth was initiated by subcutaneous administration of $5 \times 10(5)$ LLC cells and quantified every third day.

and endosialin [69] suggested parallels between the placental and tumor microenvironment, namely immune suppression, active angiogenesis, and the secretion of matrix metalloproteinase associated with metastasis, not just a functional, but also a molecular homology between placenta, tumor cells and tumor-associated endothelium. Based on the safety and possible efficacy of the Govallo vaccine, we sought to utilize placental endothelial cells as a polyvalent antigenic source for stimulation of immunity against proliferating endothelial cells that have been primed with interferon gamma to stimulate immunogenicity. We termed this product "ValloVax"

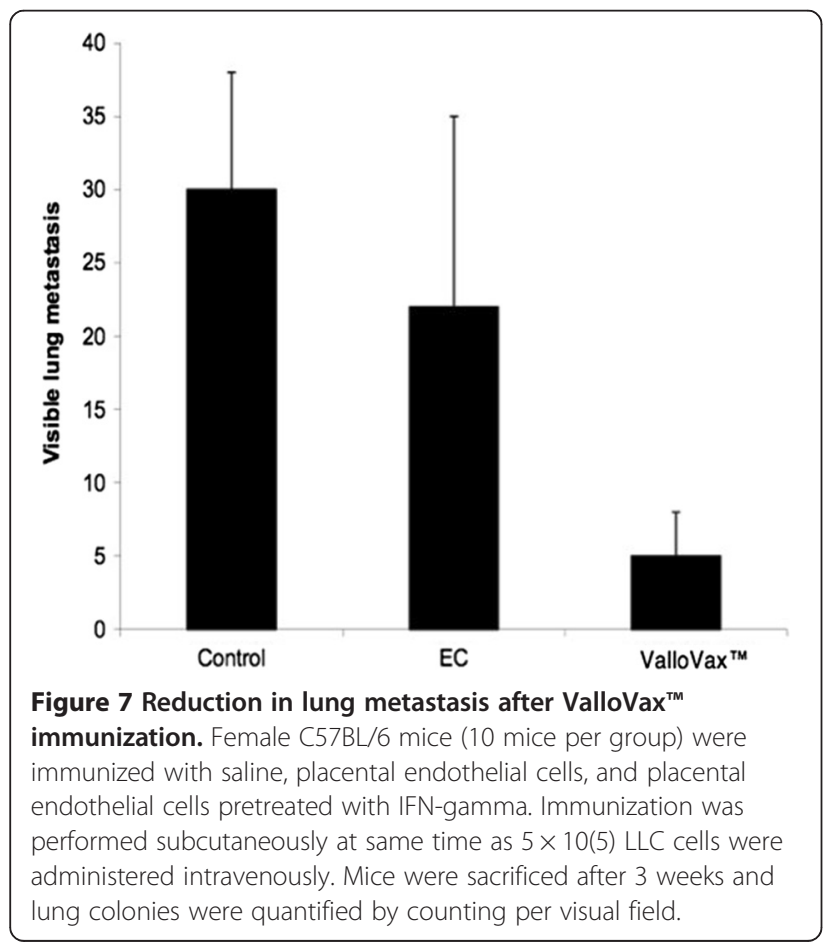

Here we demonstrate the therapeutic activity of ValloVax ${ }^{\mathrm{mm}}$ against a wide range of histologically distinct tumor types, suggesting that the effect is acting against new blood vessels and not against shared tumor antigens. Additionally, the main concern of utilization of an antiendothelial vaccine would be the possibility of inducing autoimmunity against the endothelium. This has not been observed in the 28 day safety study. We have demonstrated that tumor inhibiting activity was preserved when cells were mitotically inactivated, however was substantially reduced when cell lysate was utilized. It is important to note that adjuvants were not administered as part of the vaccination inoculum. Accordingly, manipulation of the vaccine administration either by modification of dosage or frequency may induce more potent therapeutic responses. One of the deficiencies of the current study is the lack of direct demonstration that inhibition of endothelial proliferation was responsible for reduction in tumors. Although it is unlikely that direct tumor immunity was induced to all three tumor types assessed by the administration of ValloVax ${ }^{\text {Tix }}$, this possibility cannot be excluded. Supporting the possibility that anti-endothelial immunity was induced is data demonstrating sera of immunized mice was able to inhibit proliferation of endothelial cells in vitro. These data are currently the subject of an additional manuscript looking at more detailed mechanisms of immunity and tumor biology. As well further studies on combination of ValloVax ${ }^{\text {ma }}$ with standard of care treatments will be studied.

\section{Conclusion}

Based on existing preclinical and clinical data demonstrating safety of endothelial cell vaccination, combined with the recent data described herein, ValloVax ${ }^{\mathrm{Tm}}$ appears to be a promising antiangiogenic vaccine platform. Demonstration of efficacy in 3 different animal models supports possibility utilization against a broad spectrum of tumors. 


\section{Additional file}

Additional file 1: Seven male and female mice per group were treated with control, or $500,000,2$ million or 4 million ValloVax ${ }^{\mathrm{TM}}$ irradiated cells subcutaneously. Cells were administrated on days 0,7 , 14, and 21. Body weight was assessed on days 1, 14 and 28, whereas, biochemical and hematological parameters were evaluated at the termination of the experiment on day 28.

\section{Competing interests}

TEl, HM, JSS, SCW are employees or board members of Batu Biologics.

\section{Authors' contributions}

TEI, SL, HM, YVY, JSS, ANP, SK, WM, SCW contributed to the development of the concept, experimental design, experiment execution, data analysis and writing of the manuscript. All authors have read the manuscript and agree to its submission. All authors read and approved the final manuscript.

\section{Acknowledgement}

The work in this publication was funded by Batu Biologics.

\section{Author details}

'Batu Biologics Inc, San Diego, 9255 Towne Centre Drive, Suite 450, San Diego, CA 92121, USA. ²Department of Endocrinology, The Affiliated Zhongshan Hospital of Dalian University, Dalian 116001, China. ${ }^{3}$ Nova Southeastern University, Fort Lauderdale, Florida, USA. ${ }^{4}$ Department of Surgery, University of Utah, Salt Lake City, Utah. ${ }^{5}$ Department of Neurosciences, University of California San Diego, 9500 Gilman Dr., MSC 0752, La Jolla, San Diego, CA 92093-0752, USA. ${ }^{6}$ Translational Neuro-Oncology Laboratories, Moores Cancer Center, University of California San Diego, 3855 Health Sciences Dr., MSC 0819, La Jolla, San Diego, CA 92093-0819, USA. 7Department of Immunology, University of Western Ontario, London, Ontario, Canada.

Received: 27 January 2015 Accepted: 18 February 2015

Published online: 14 March 2015

\section{References}

1. Kmieciak M, Payne KK, Idowu MO, Grimes MM, Graham L, Ascierto ML, et al. Tumor escape and progression of HER-2/neu negative breast cancer under immune pressure. J Transl Med. 2011;9:35.

2. Beatty $G L$, Paterson $Y$. IFN-gamma can promote tumor evasion of the immune system in vivo by down-regulating cellular levels of an endogenous tumor antigen. J Immunol. 2000;165(10):5502-8.

3. Kmieciak M, Knutson $\mathrm{KL}$, Dumur $\mathrm{Cl}$, Manjili MH. HER-2/neu antigen loss and relapse of mammary carcinoma are actively induced by T cell-mediated anti-tumor immune responses. Eur J Immunol. 2007;37(3):675-85.

4. Chang CC, Campoli M, Restifo NP, Wang X, Ferrone S. Immune selection of hot-spot beta 2-microglobulin gene mutations, HLA-A2 allospecificity loss, and antigen-processing machinery component down-regulation in melanoma cells derived from recurrent metastases following immunotherapy. J Immunol. 2005;174(3):1462-71.

5. Marincola FM, Jaffee EM, Hicklin DJ, Ferrone S. Escape of human solid tumors from T-cell recognition: molecular mechanisms and functional significance. Adv Immunol. 2000;74:181-273.

6. Hicklin DJ, Wang Z, Arienti F, Rivoltini L, Parmiani G, Ferrone S. beta2-Microglobulin mutations, HLA class I antigen loss, and tumor progression in melanoma. J Clin Invest. 1998;101(12):2720-9.

7. Lampson LA, Fisher CA, Whelan JP. Striking paucity of HLA-A, B, C and beta 2-microglobulin on human neuroblastoma cell lines. J Immunol. 1983;130 (5):2471-8.

8. Yan WH. HLA-G expression in cancers: potential role in diagnosis, prognosis and therapy. Endocr Metab Immune Disord Drug Targets. 2011;11(1):76-89.

9. Seliger B. B7-H abnormalities in melanoma and clinical relevance. Methods Mol Biol. 2014;1102:367-80.

10. Rutkowski MR, Stephen TL, Conejo-Garcia JR. Anti-tumor immunity: myeloid leukocytes control the immune landscape. Cell Immunol. 2012;278(1-2):21-6.

11. Carmeliet $P$, Jain RK. Angiogenesis in cancer and other diseases. Nature. 2000;407(6801):249-57.
12. Folkman J. The role of angiogenesis in tumor growth. Semin Cancer Biol. 1992;3(2):65-71.

13. Motz GT, Santoro SP, Wang LP, Garrabrant T, Lastra RR, Hagemann IS, et al. Tumor endothelium FasL establishes a selective immune barrier promoting tolerance in tumors. Nat Med. 2014;20(6):607-15.

14. Facciponte JG, Ugel S, De Sanctis F, Li C, Wang L, Nair G, et al. Tumor endothelial marker 1-specific DNA vaccination targets tumor vasculature. J Clin Invest. 2014;124(4):1497-511.

15. Meng MB, Jiang XD, Deng L, Na FF, He JZ, Xue JX, et al. Enhanced radioresponse with a novel recombinant human endostatin protein via tumor vasculature remodeling: experimental and clinical evidence. Radiother Oncol. 2013;106(1):130-7.

16. Czarnota GJ, Karshafian R, Burns PN, Wong S, Al Mahrouki A, Lee JW, et al. Tumor radiation response enhancement by acoustical stimulation of the vasculature. Proc Natl Acad Sci U S A. 2012;109(30):E2033-41.

17. Peng F, Xu Z, Wang J, Chen Y, Li Q, Zuo Y, et al. Recombinant human endostatin normalizes tumor vasculature and enhances radiation response in xenografted human nasopharyngeal carcinoma models. PLoS One. 2012;7(4):e34646

18. Zawaski JA, Gaber MW, Sabek OM, Wilson CM, Duntsch CD, Merchant TE. Effects of irradiation on brain vasculature using an in situ tumor model. Int J Radiat Oncol Biol Phys. 2012;82(3):1075-82.

19. Truman JP, García-Barros M, Kaag M, Hambardzumyan D, Stancevic B, Chan M et al. Endothelial membrane remodeling is obligate for anti-angiogenic radiosensitization during tumor radiosurgery. PLoS One. 2010;19;5(8):e12310.

20. Okaji Y, Tsuno NH, Tanaka M, Yoneyama S, Matsuhashi M, Kitayama J, et al. Pilot study of anti-angiogenic vaccine using fixed whole endothelium in patients with progressive malignancy after failure of conventional therapy. Eur J Cancer. 2008;44(3):383-90.

21. Brose K, Bland KS, Wang KH, Arnott D, Henzel W, Goodman CS, et al. Slit proteins bind Robo receptors and have an evolutionarily conserved role in repulsive axon guidance. Cell. 1999:96(6):795-806.

22. Yadav SS, Narayan G. Role of ROBO4 signalling in developmental and pathological angiogenesis. Biomed Res Int. 2014;2014:683025.

23. Zhuang X, Ahmed F, Zhang Y, Ferguson HJ, Steele JC, Steven NM, et al. Robo4 vaccines induce antibodies that retard tumor growth. Angiogenesis. 2014;18(1):83-95.

24. Paprocka M, Krawczenko A, Dus D, Kantor A, Carreau A, Grillon C, et al. CD133 positive progenitor endothelial cell lines from human cord blood. Cytometry A. 2011;79(8):594-602

25. Chen C, Zeng L, Ding S, Xu K. Adult endothelial progenitor cells retain hematopoiesis potential. Transplant Proc. 2010:42(9):3745-9.

26. Smadja DM, Bièche I, Helley D, Laurendeau I, Simonin G, Muller L, et al. Increased VEGFR2 expression during human late endothelial progenitor cells expansion enhances in vitro angiogenesis with up-regulation of integrin alpha(6). J Cell Mol Med. 2007;11(5):1149-61.

27. Rafii S, Avecilla S, Shmelkov S, Shido K, Tejada R, Moore MA, et al. Angiogenic factors reconstitute hematopoiesis by recruiting stem cells from bone marrow microenvironment. Ann N Y Acad Sci. 2003:996:49-60.

28. Hattori K, Heissig B, Wu Y, Dias S, Tejada R, Ferris B, et al. Placental growth factor reconstitutes hematopoiesis by recruiting VEGFR1(+) stem cells from bone-marrow microenvironment. Nat Med. 2002;8(8):841-9.

29. Ziegler BL, Valtieri M, Porada GA, De Maria R, Müller R, Masella B, et al. KDR receptor: a key marker defining hematopoietic stem cells. Science. 1999;285(5433):1553-8

30. Yan HX, Cheng P, Wei HY, Shen GB, Fu LX, Ni J, et al. Active immunotherapy for mouse breast cancer with irradiated whole-cell vaccine expressing VEGFR2. Oncol Rep. 2013;29(4):1510-6.

31. Feng $\mathrm{K}$, Zhao H, Chen J, Yao D, Jiang X, Zhou W, et al. Anti-angiogenesis effect on glioma of attenuated Salmonella typhimurium vaccine strain with flk-1 gene. J Huazhong Univ Sci Technolog Med Sci. 2004;24(4):389-91.

32. Wada S, Tsunoda T, Baba T, Primus FJ, Kuwano H, Shibuya M, et al. Rationale for antiangiogenic cancer therapy with vaccination using epitope peptides derived from human vascular endothelial growth factor receptor 2. Cancer Res. 2005;65(11):4939-46.

33. Yan J, Jia $R$, Song $H$, Liu $Y$, Zhang $L$, Zhang $W$, et al. A promising new approach of VEGFR2-based DNA vaccine for tumor immunotherapy. Immunol Lett. 2009;126(1-2):60-6.

34. Ren S, Fengyu, Zuo S, Zhao M, Wang X, Wang X et al. Inhibition of tumor angiogenesis in lung cancer by T4 phage surface displaying mVEGFR2 vaccine. Vaccine. 2011;29(34):5802-11. 
35. Wei Y, Sun Y, Song C, Li H, Li Y, Zhang K, et al. Enhancement of DNA vaccine efficacy by targeting the xenogeneic human chorionic gonadotropin, survivin and vascular endothelial growth factor receptor 2 combined tumor antigen to the major histocompatibility complex class II pathway. J Gene Med. 2012;14(5):353-62.

36. Suzuki H, Fukuhara M, Yamaura T, Mutoh S, Okabe N, Yaginuma H, et al. Multiple therapeutic peptide vaccines consisting of combined novel cancer testis antigens and anti-angiogenic peptides for patients with non-small cell lung cancer. J Transl Med. 2013;11:97.

37. Lladser $A$, Ljungberg $K$, Tufvesson $H$, Tazzari $M$, Roos AK, Quest AF, et al. Intradermal DNA electroporation induces survivin-specific CTLs, suppresses angiogenesis and confers protection against mouse melanoma. Cancer Immunol Immunother. 2010;59(1):81-92.

38. Xiang R, Luo Y, Niethammer AG, Reisfeld RA. Oral DNA vaccines target the tumor vasculature and microenvironment and suppress tumor growth and metastasis. Immunol Rev. 2008;222:117-28.

39. Xiang R, Mizutani N, Luo Y, Chiodoni C, Zhou H, Mizutani M. A DNA vaccine targeting survivin combines apoptosis with suppression of angiogenesis in lung tumor eradication. Cancer Res. 2005;65(2):553-61.

40. Plum SM, Vu HA, Mercer B, Fogler WE, Fortier AH. Generation of a specific immunological response to FGF-2 does not affect wound healing or reproduction. Immunopharmacol Immunotoxicol. 2004;26(1):29-41.

41. Wei YQ, Huang MJ, Yang L, Zhao X, Tian L, Lu Y, et al. Immunogene therapy of tumors with vaccine based on Xenopus homologous vascular endothelial growth factor as a model antigen. Proc Natl Acad Sci U S A. 2001;98(20):11545-50.

42. Liu JY, Wei YQ, Yang L, Zhao X, Tian L, Hou JM, et al. Immunotherapy of tumors with vaccine based on quail homologous vascular endothelial growth factor receptor-2. Blood. 2003;102(5):1815-23.

43. Su JM, Wei YQ, Tian L, Zhao X, Yang L, He QM, et al. Active immunogene therapy of cancer with vaccine on the basis of chicken homologous matrix metalloproteinase-2. Cancer Res. 2003;63(3):600-7.

44. Tan GH, Wei YQ, Tian L, Zhao X, Yang L, Li J, et al. Active immunotherapy of tumors with a recombinant xenogeneic endoglin as a model antigen. Eur J Immunol. 2004;34(7):2012-21.

45. Jiao JG, Li YN, Wang H, Liu Q, Cao JX, Bai RZ, et al. A plasmid DNA vaccine encoding the extracellular domain of porcine endoglin induces anti-tumour immune response against self endoglin-related angiogenesis in two liver cancer models. Dig Liver Dis. 2006;38(8):578-87.

46. Natarajan M, Gibbons CF, Mohan S, Moore S, Kadhim MA. Oxidative stress signalling: a potential mediator of tumour necrosis factor alpha-induced genomic instability in primary vascular endothelial cells. Br J Radiol. 2007;80(Spec No 1):S13-22.

47. Hida K, Ohga N, Akiyama K, Maishi N, Hida Y. Heterogeneity of tumor endothelial cells. Cancer Sci. 2013;104(11):1391-5.

48. Wei YQ, Wang QR, Zhao X, Yang L, Tian L, Lu Y, et al. Immunotherapy of tumors with xenogeneic endothelial cells as a vaccine. Nat Med. 2000;6(10):1160-6.

49. Scappaticci FA1, Contreras A, Boswell CA, Lewis JS, Nolan G. Polyclonal antibodies to xenogeneic endothelial cells induce apoptosis and block support of tumor growth in mice. Vaccine. 2003;21(19-20):2667-77.

50. Tanaka M, Tsuno NH, Fujii T, Todo T, Saito N, Takahashi K. Human umbilical vein endothelial cell vaccine therapy in patients with recurrent glioblastoma. Cancer Sci. 2013;104(2):200-5.

51. Xu M, Zhou L, Zhang P, Lu Y, Ge C, Yao W, et al. Enhanced antitumor efficacy by combination treatment with a human umbilical vein endothelial cell vaccine and a tumor cell lysate-based vaccine. Tumour Biol. 2013;34(5):3173-82.

52. Ferguson HJ, Wragg J, Ismail T, Bicknell R. Vaccination against tumour blood vessels in colorectal cancer. Eur J Surg Oncol. 2014;40(2):133-6.

53. Zhang W, Liu JN, Tan XY. Vaccination with xenogeneic tumor endothelial proteins isolated in situ inhibits tumor angiogenesis and spontaneous metastasis. Int J Cancer. 2009;125(1):124-32.

54. Yoshiura K, Nishishita T, Nakaoka T, Yamashita N, Yamashita N. Inhibition of B16 melanoma growth and metastasis in C57BL mice by vaccination with a syngeneic endothelial cell line. J Exp Clin Cancer Res. 2009;28:13.

55. Okaji Y, Tsuno NH, Saito S, Yoneyama S, Tanaka M, Nagawa H, et al. Vaccines targeting tumour angiogenesis-a novel strategy for cancer immunotherapy. Eur J Surg Oncol. 2006;32(4):363-70.

56. Ugele B, Lange F. Isolation of endothelial cells from human placental microvessels: effect of different proteolytic enzymes on releasing endothelial cells from villous tissue. In Vitro Cell Dev Biol Anim. 2001;37(7):408-13.

57. Kacemi A, Challier JC, Galtier M, Olive G. Isolation of villous microvessels from the human placenta. C R Acad Sci III. 1997;320(2):171-7.

58. Moscatelli DA, Presta M, Mignatti P, Mullins DE, Crowe RM, Rifkin DB. Purification and biological activities of an angiogenesis factor from human placenta. Anticancer Res. 1986;6(4):861-3.

59. Takenaga K. Angiogenic signaling aberrantly induced by tumor hypoxia. Front Biosci (Landmark Ed). 2011;16:31-48.

60. Folkman J, Long Jr DM, Becker FF. Growth and metastasis of tumor in organ culture. Cancer. 1963;16:453-67.

61. de Castro JG, Puglisi F, de Azambuja E, El Saghir NS, Awada A. Angiogenesis and cancer: a cross-talk between basic science and clinical trials (the "do ut des" paradigm). Crit Rev Oncol Hematol. 2006;59(1):40-50.

62. Patra D, Sandell LJ. Antiangiogenic and anticancer molecules in cartilage. Expert Rev Mol Med. 2012;19;14:e10.

63. Culy C. Bevacizumab: antiangiogenic cancer therapy. Drugs Today (Barc). 2005;41(1):23-36

64. Vaklavas C, Lenihan D, Kurzrock R, Tsimberidou AM. Anti-vascular endothelial growth factor therapies and cardiovascular toxicity: what are the important clinical markers to target? Oncologist. 2010;15(2):130-41.

65. Kerbel RS. Reappraising antiangiogenic therapy for breast cancer. Breast. 2011;20 Suppl 3:S56-60.

66. Harandi A. Immunoplacental therapy, a potential multi-epitope cancer vaccine. Med Hypotheses. 2006;66(6):1182-7.

67. Yoshikawa M, Mukai Y, Okada Y, Tsumori Y, Tsunoda S, Tsutsumi Y, et al Robo4 is an effective tumor endothelial marker for antibody-drug conjugates based on the rapid isolation of the anti-Robo4 cell-internalizing antibody. Blood. 2013;121(14):2804-13.

68. Mura M, Swain RK, Zhuang X, Vorschmitt H, Reynolds G, Durant S, et al. Identification and angiogenic role of the novel tumor endothelial marker CLEC14A. Oncogene. 2012;31(3):293-305.

69. Simonavicius N, Robertson D, Bax DA, Jones C, Huijbers IJ, Isacke CM Endosialin (CD248) is a marker of tumor-associated pericytes in high-grade glioma. Mod Pathol. 2008;21(3):308-15.

\section{Submit your next manuscript to BioMed Central and take full advantage of:}

- Convenient online submission

- Thorough peer review

- No space constraints or color figure charges

- Immediate publication on acceptance

- Inclusion in PubMed, CAS, Scopus and Google Scholar

- Research which is freely available for redistribution 